\title{
Pediatric Inflammatory Multisystem Syndrome Temporally Associated with SARS-CoV-2
}

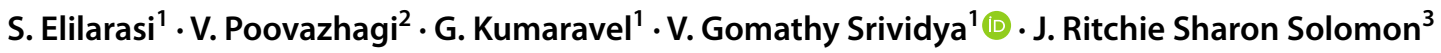

Received: 23 January 2021 / Accepted: 7 July 2021 / Published online: 24 November 2021

(c) Dr. K C Chaudhuri Foundation 2021

\begin{abstract}
Objectives To know the clinical presentation and outcome of children with pediatric inflammatory multisystem syndrome temporally associated with SARS-CoV- 2 (PIMS-TS) at a pediatric tertiary care center in Chennai.

Methods Clinical and biochemical parameters of 65 children with PIMS-TS treated between July and October 2020 were studied. All children had their COVID RT-PCR and IgG COVID antibodies tests done.

Results Mean age of the study group was $5.65 \pm 3.68 \mathrm{y}$. Fever with red eyes, rash, vomiting, abdominal pain, and shock were common presenting features. Sixty percent of the study group had Kawasaki/incomplete Kawasaki features. Sixty-seven percent of the study group had coronary dilatation, $41 \%$ presented with shock, and $25 \%$ had left ventricular dysfunction. Coronary aneurysms were documented in $58 \%$ of the study group ( $z$ score more than 2.5 ). Respiratory presentation with pneumonia was seen in $10 \%$. Four children presented with acute abdomen. Acute kidney injury, acute liver failure, hemolysis, pancytopenia, macrophage activation syndrome, encephalopathy, and multiorgan dysfunction syndrome (MODS) were other features. Forty-three percent required noninvasive oxygen support and $15.4 \%$ required mechanical ventilation. Intravenous immunoglobulin (73.8\%) and methylprednisolone (49.8\%) were used for therapy. Mortality in the study was 6\%, which was due to MODS.

Conclusions Acute febrile illness with mucocutaneous and gastrointestinal manifestations should have PIMS-TS as a possible differential diagnosis and needs evaluation with inflammatory markers and SARS-CoV-2 antibodies.
\end{abstract}

Keywords PIMS-TS $\cdot$ Kawasaki syndrome $\cdot$ Macrophage activation syndrome $\cdot$ COVID-19 $\cdot$ MIS-C

\section{Introduction}

Since the pandemic of coronavirus disease 2019 (COVID19), many countries around the world have reported their data but data from India have been limited. Initial reports suggested that children were spared from severe disease [1]. However, currently there are increasing reports of pediatric multisystem inflammatory syndrome temporally associated

V. Gomathy Srividya gomathysrividya@gmail.com

1 Department of Pediatrics, Institute of Child Health and Hospital for Children, Chennai, Tamil Nadu 600008, India

2 HOD \& Professor, Department of Pediatric Intensive Care, Institute of Child Health and Hospital for Children, Chennai, Tamil Nadu, India

3 Department of Pediatric Cardiology, Institute of Child Health and Hospital for Children, Chennai, Tamil Nadu, India with SARS-CoV-2 (PIMS-TS) also called the multisystem inflammatory syndrome in children and adolescents temporally related to COVID-19 and the multisystem inflammatory syndrome in children (MIS-C) [2, 3]. PIMS-TS is a spectrum of disease with phenotypes including Kawasaki disease, toxic shock syndrome, and macrophage activation syndrome $[4,5]$. The authors describe their experience of treating children with PIMS-TS from a pediatric tertiary care center in South India.

\section{Material and Methods}

This was a descriptive study undertaken at the pediatric tertiary care center at Chennai and all children with PIMSTS admitted to the hospital between July and October 2020 were included. Their medical and laboratory data were analyzed and the symptomatology and outcome were reported. The criteria for diagnosis was based on Royal College of 
Pediatrics and Child Health, United Kingdom for PIMS-TS [2]. Inclusion criteria included child presenting with:

- Persistent fever $>38.5^{\circ} \mathrm{C}$

- Laboratory evidence of inflammation (ex: neutrophilia, elevated CRP, lymphopenia)

- Single or multiorgan dysfunction

Exclusion of alternative causes. SARS-CoV-2 testing can be positive or negative.

The primary aim of this study was to identify the different phenotypes of PIMS-TS spectrum and its outcome. Children were included after caregiver's informed consent. Data on demographics, clinical presentation, laboratory, and radiological investigation, ECG, ECHO, and management and outcome were collected. Vital parameters like heart rate, respiratory rate, and blood pressure were analyzed as per standard definitions [6]. Echocardiogram was done by a pediatric cardiologist using GE VIVID S6 ultrasound machine with phased array cardiac transducer. Coronary abnormalities were categorized as normal if $z$ score was $<2$, dilatation, if between 2 and 2.5 , mild aneurysm if between 2.5 and 5, moderate if between 5 and 10, and severe if $>10$ [7]. SARS-CoV-2 IgG antibody test was performed with ICMR-approved SARS-CoV-2 IgG antibody titer assay kits as per manufacturer's instructions; and titers more than 10 AU was considered reactive. SARS-CoV-2 real-time polymerase chain reaction (RT-PCR) was done by ICMR-approved laboratory. All children were followed up till discharge from the hospital or death in case of hospital mortality. The study was undertaken after ethics approval. Statistical analysis was undertaken using the Epi Info version 7.2 stat software. Continuous variables were reported as means, medians, and range and categorical variables as frequency.

\section{Results}

A total of 65 children were included for analysis in the study. The age group ranged from 2 mo to $12 \mathrm{y}$ with a median age of $5 \mathrm{y}$, of which, $6.1 \%$ (4/65) were less than $12 \mathrm{mo}, 44.6 \%$ $(29 / 65)$ were between 1 and $5 \mathrm{y}$, and $49.23 \%(32 / 65)$ were between 5 and $12 \mathrm{y}$. Male to female ratio was 1.5:1. None of these children were obese. Nine (6\%) children had history of contact with individuals with COVID-19 infection and 2 of them were COVID-19 RT-PCR-positive in the previous 4 wk. Two children had no history of contact or previous COVID infection or presence of antibody, but had features of coronary aneurysm. SARS-CoV-2 IgG antibodies were present in $86 \%(56 / 65)$.

The median duration of illness was $5 \mathrm{~d}$ (range 2-14 d). Of the 65 children, 27 (41.5\%) presented with shock at the emergency room and 17 (26\%) were hypotensive at admission to PICU. Thirty-three children required PICU care. Inotrope support was needed in 25/65 (38.5\%) children. Twelve children required one inotrope, 9 required two inotropes, 3 children required three inotropes, and 1 child required four inotropes. Clinical features are summarized in Table 1. Differential diagnosis considered at admission included acute febrile illness, dengue, scrub typhus fever, and fever with thrombocytopenia, bronchopneumonia, and acute abdomen. Highest temperature recorded was $108^{\circ} \mathrm{F}$ with a mean of $102.58 \pm 1.72{ }^{\circ} \mathrm{F}$. Respiratory involvement was in the form of breathlessness, pleural effusion $12 \%$ (8/65), consolidation in $9 \%(6 / 65)$, and ARDS in 1 child. Nearly half of the children [54\% (38/65)] required respiratory support. Twentythree needed non-rebreathing mask oxygen, 3 required highflow humidified nasal cannula oxygen (HHFNC), 2 required BIPAP, and 10 required mechanical ventilation.

Of the 65 children, 35 had received either antibiotics or antiemetics or analgesics/antipyretics before presenting to the authors' institute. Clinical presentation was predominantly acute febrile illness with mucocutaneous and gastrointestinal manifestations as shown in Table 1. Mucocutaneous features included skin rash, edema, conjunctival congestion, cracked lips, red lips, red tongue, and peeling of skin. Gastrointestinal manifestations included, nausea, vomiting, diarrhea, and pain in abdomen. Cardiac involvement included coronary artery abnormalities $(68 \%, 44 / 65)$, tachycardia $(60 \%, 39 / 65)$, shock $(42 \%, 27 / 65)$, hypotension $(26 \%$, $17 / 65)$, and left ventricular dysfunction $(25 \%, 16 / 65)$. Coronary artery abnormalities seen were nontapering coronaries, echogenic coronaries, coronary dilatation, and coronary aneurysm with a $z$ score $>2.5$. Coronary abnormalities were seen in 44 children. Eight had dilatation ( $z$ score 2-2.5), 34 had small aneurysm ( $z$ score $2.5-5), 1$ had medium aneurysm ( $z$ score 5-10), and 1 had echogenic and tram tracking of coronaries. Figure 1 shows dilated coronaries in short

Table 1 Clinical presentation $(n=65)$

\begin{tabular}{llll}
\hline Presentation & $n(\%)$ & Presentation & $n(\%)$ \\
\hline Fever at admission & $63(96.9)$ & Nausea & $15(23.08)$ \\
Vomiting & $47(72.3)$ & Myalgia & $14(21.54)$ \\
Conjunctivitis & $45(69.23)$ & Headache & $8(12.5)$ \\
Edema & $36(55.38)$ & Sore throat & $6(9.28)$ \\
Rash & $35(53.85)$ & Skin peeling & $6(9.23)$ \\
Diarrhea & $29(44.62)$ & Bleeds & $3(4.52)$ \\
Shortness of breath & $21(32.3)$ & Chest pain & $2(3.13)$ \\
Abdominal pain & $29(49.62)$ & Seizures & $2(3)$ \\
Red lips & $20(30.7)$ & & \\
Altered sensorium & $19(29.23)$ & & \\
Reduced urine output & $18(27.69)$ & & \\
Red tongue & $15(29.23)$ & & \\
\hline
\end{tabular}




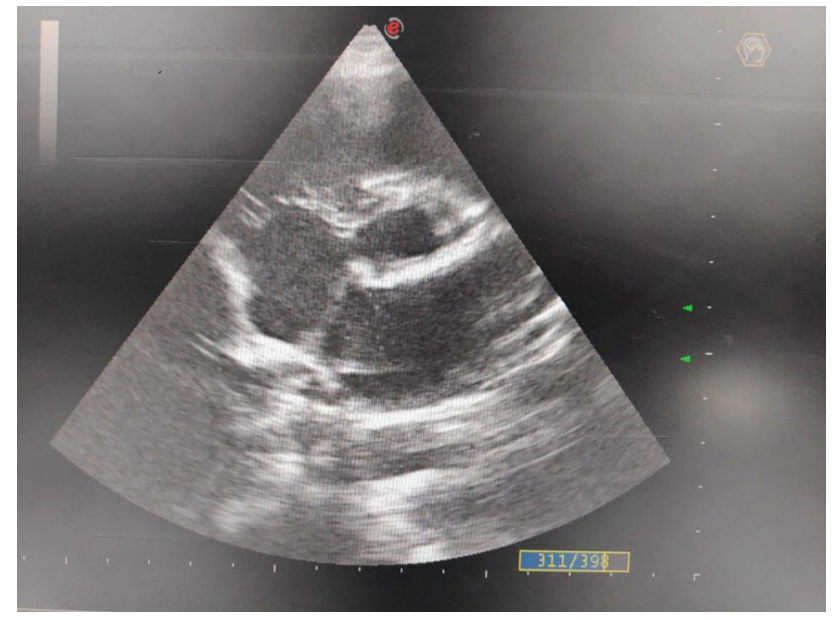

Fig. 1 Echo image of dilated left coronary in short axis view

axis view. With respect to 43 children with coronary dilatation and aneurysm, factors like age, gender, clinical features, shock, AKI, MODS, HLH, and left ventricular dysfunction did not reveal significant difference in those without coronary dilatation. However, the group with coronary dilatation had significantly higher NLR ratio ( $p=0.014)$.

Other ECHO findings included pericardial effusion (49.2\%), mitral regurgitation (17/65), aortic regurgitation $(2 / 65)$, and tricuspid regurgitation (3/65). One child had supraventricular tachycardia. Neurological features included encephalopathy in 6\% (4/65), seizures in 3\% (2/65), and stroke in 1 child. One child had refractory hypertension for weeks following diagnosis and is on antihypertensives. Acute abdomen (surgical presentation) was seen in 4 children. Three of them were operated and were later diagnosed as PIMS-TS. Associated comorbid conditions included febrile seizures $(3 / 65)$, children known to have reactive airway disease (3/65), and acute lymphoblastic leukemia (2/65). Hematological abnormalities included anemia (78\%, $51 / 65)$, neutrophilic leucocytosis $(70.7 \%, 46 / 65)$, lymphopenia $(72.3 \%, 47 / 65)$, high neutrophil lymphocyte ratio more than $3(55 \%, 33 / 65)$, thrombocytopenia $(55 \%, 33 / 65)$, thrombocytosis $(31.25 \%, 20 / 65)$, pancytopenia $(29 \%, 19 / 65)$, and hemophagocytosis $(6 \%, 4 / 65)$. Renal involvement with elevated urea was reported in $37 \%$ (24/65) of children. Renal complications (pRIFLE) included risk in 4 children, injury in 5, and failure in 3. Hepatic involvement included elevated SGOT (49\%, 32/65), elevated SGPT (32\%, 21/65), and hyperbilirubinemia $(17 \%, 11 / 65)$, ascites in $15 \%(10 / 65)$, and acute liver cell failure in $7.7 \%$ (5/65). Other laboratory abnormalities included hyponatremia $(84.62 \%, 55 / 65)$ and hypoalbuminemia $(40 \%, 26 / 65)$. Thirty-eight children had LDH levels measured and all of them had elevated LDH and $81.6 \%$ (31/38) had anemia with elevated LDH levels.
Coinfections noted were dengue, scrub typhus, and typhoid in 1 child each, which were excluded from the study group.

Overall, the clinical phenotypes were as follows: Kawasaki phenotype $[51 / 65,(78.4 \%)]$, toxic shock syndrome phenotype [27/65, (41\%)], and HLH phenotype [6/65, (9.23\%)]. Nine children (13.8\%) had no specific phenotype. Multiorgan failure was seen in $7(10.7 \%)$ children. The laboratory findings are summarized in Table 2. Management of children with coronary dilatation was by $2 \mathrm{~g} / \mathrm{kg}$ of intravenous immunoglobulin (IVIG) and aspirin with or without steroids. Children with LV dysfunction and those refractory to IVIG were on pulse methylprednisolone. Presence of myocardial dysfunction or hemodynamic compromise in the form of shock were considered as upfront indications to start on methylprednisolone along with IVIG. Therapy included IVIG and methylprednisolone in 36 children, IVIG alone in 12 children, methyl prednisolone alone in 6 , dexamethasone in 3 children, tocilizumab in 4 children, and aspirin in 48 children. Overall mortality was 6\% (4/65); among those who died, all 4 received IVIG and methyl prednisolone, and 1 received tocilizumab in addition. All the 4 children who died had shock, multiorgan dysfunction (MODS), encephalopathy, and macrophage activation syndrome (MAS). Three had Kawasaki-like features and 3 had acute kidney injury (AKI). High NLR ratio, AKI, MODS, shock, and HLH were identified to be associated with mortality with a $p$ value of $0.00002,0.008,0.0001,0.013$, and 0.0008 , respectively (Table 3).

\section{Discussion}

PIMS-TS is a rare pediatric condition characterized by hyperinflammation and multiorgan dysfunction frequently following an infectious trigger by SARS-CoV-2. PIMS-TS was initially reported by pediatricians in UK. Subsequently, the US center for disease control and the WHO published the criteria and named it as multisystem inflammatory syndrome in children (MIS-C) and RCPCH defined it as PIMS-TS [2, 3]. This condition is typically seen $2-4$ wk after an acute COVID-19 infection. However, in the present cohort, only 9 children reported history of COVID-19 infection or contact with COVID infection in the recent past. The median age group was similar to other published reports [5, 8, 9] and as described in other reports, a male preponderance was found in this study as well [10]. Data from the present study add to the evidence that a large proportion of children with PIMS-TS present with gastrointestinal manifestations [8, 11-15], thus highlighting that PIMS-TS should be considered in children presenting with fever and GI symptoms. Mucocutaneous features like red eye and rashes is another common presentation similar to the studies elsewhere [16, 17]. Median duration of illness was $5 \mathrm{~d}$ and 
Table 2 Laboratory parameters in PIMS-TS $(n=65)$

Parameter - range (mean \pm SD)

Total count (cells/cumm)

$3200-42,000(12,386 \pm 6594.7)$

Hemoglobin $(\mathrm{g} / \mathrm{dL})$

$5-16(9.92 \pm 1.8)$

Ferritin (ng/mL)

Initial 32-10,379 $(965 \pm 1567)$

Highest 90-11,600 (1237 \pm 2752$)$

Triglycerides (mg/dL) 44-509 (64.86 \pm 70$)$

Prothrombin time (second) 10.5-34.6 (16.42 \pm 5.2$)$

aPTT (second) 13.2-65.1 (32.3 \pm 10.19$)$

INR0.75-2.33 (1.244 \pm 0.378$)$

\author{
Sodium $(\mathrm{mEq} / \mathrm{L})$ \\ Potassium $(\mathrm{mEq} / \mathrm{L})$ \\ Urea (mg/dL) \\ Initial 8-145 (32.92 \pm 29.45$)$ \\ Highest $8-210(49 \pm 49.5)$ \\ Creatinine $(\mathrm{mg} / \mathrm{dL})$ \\ Initial $0.2-2.6(0.52 \pm 0.37)$ \\ Highest $0.3-4.8(0.695 \pm 0.77)$
}

$118-142(131.2 \pm 4.3)$

$2.8-6.1(4.40 \pm 0.67)$

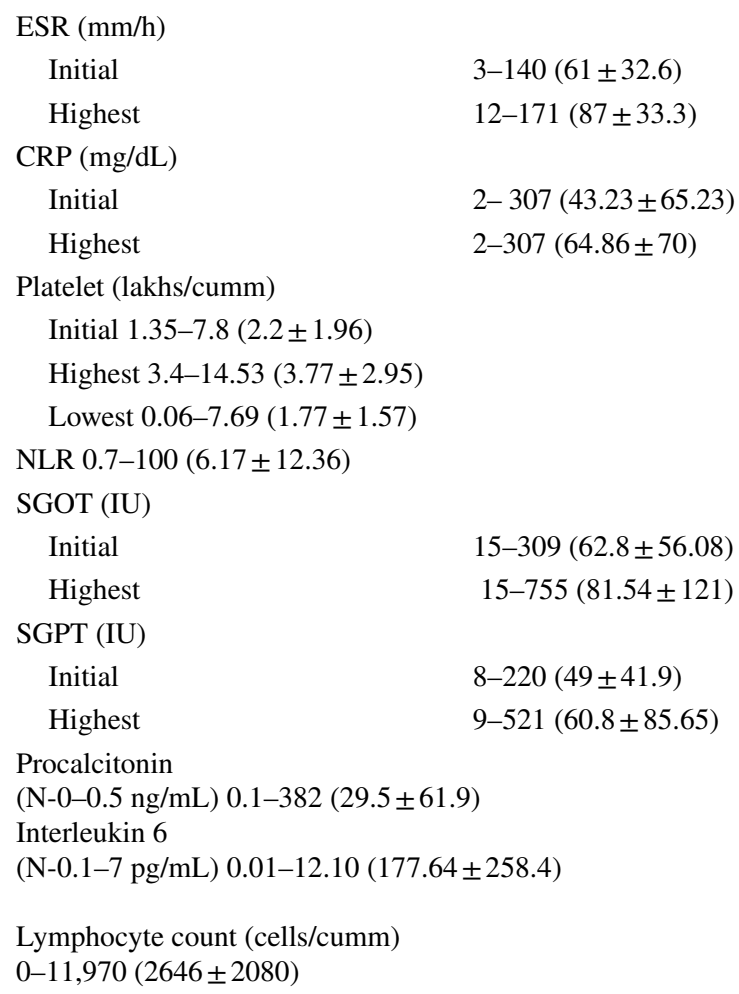

Table 3 Comparison between survivors and nonsurvivors

\begin{tabular}{llll}
\hline & $N=61$ & $N=4$ & $p$ value \\
\hline Shock & 23 & 4 & 0.013 \\
MODS & 3 & 4 & 0.001 \\
Coagulopathy & 8 & 3 & 0.059 \\
MAS/HLH & 3 & 3 & 0.000 \\
KD & 48 & 3 & 0.862 \\
AKI & 2 & 3 & 0.000 \\
NLR (mean) & 30.1 & 4.5 & 0.000 \\
\hline
\end{tabular}

$A K I$ Acute kidney injury; KD Kawasaki disease; MAS Macrophage activation syndrome; MODS Multiorgan dysfunction

can range from 3-9 d [5, 18]. Lymphopenia was seen in 72\% and is a common finding in PIMS-TS [12, 19]. The coronary dilatation among children with PIMS-TS varies from 8\%-63\% $[9,20,21]$. In contrast to the published literature from South India [6], more than two-thirds $(68 \%, 44 / 65)$ of the children presented with coronary abnormalities. Higher percentage of coronary involvement or the Kawasaki-like feature in this study group could be attributed to the delayed referral pattern seen during the COVID-19 pandemic. Acute abdominal pain presenting as a surgical emergency has been previously reported in children with PIMS-TS [22, 23]. Similar to this, 3 of the children in the present study were also operated for appendicitis and later diagnosed as PIMS-TS. Clinical features of PIMS-TS often overlap with the clinical presentation of other infectious diseases, especially scrub typhus fever and dengue. Hence, it is imperative to consider and exclude other causes of acute febrile illness in children in tropical countries. Presence of lymphopenia, high neutrophil -to-lymphocyte ratio, raised ESR, hyponatremia, hypoalbuminemia along with either previous history of COVID-19 or contact with COVID19 or a positive IgG antibody status would help clinicians differentiate PIMS TS.

The limitation of this study is that COVID RT-PCR-positive children were treated in COVID-exclusive facility and could not be included. Overlap of Kawasaki disease in Kawasaki phenotype of MIS-C is possible. Likewise, being a tertiary care referral hospital, children with severe complications may have been received and mild cases of PIMS-TS could have been missed or not referred to. Being a new disease there is an urgent need for further research and long-term follow-up of these children. Future research 
should focus on identifying the variables that can prognosticate which pediatric COVID-19 patients will develop MIS-C and which, if any, markers correlate with systemic outcomes.

\section{Conclusions}

Acute febrile illness with mucocutaneous and gastrointestinal manifestations should have evaluation with laboratory markers, ECHO, and SARS-CoV-2 antibodies. PIMS-TS spectrum in children includes acute febrile illness with Kawasaki-like features with or without shock, and acute abdomen. Myocardial dysfunction, shock, leucopenia, thrombocytopenia, high neutrophil-to-lymphocyte ratio, hyponatremia, and hypoalbuminemia are frequently encountered in KD phenotype PIMS-TS. Macrophage activation syndrome, encephalopathy, stroke, acute abdomen, hemolysis, and thrombotic microangiopathy are less common presentations of PIMS-TS in children. Therapy includes intravenous immunoglobulin, methylprednisolone, and immunomodulatory agents like tocilizumab. Outcome is good if recognized early and treated appropriately. High NLR ratio, AKI, MODS, shock, and HLH were identified to be associated with mortality with a $p$ value of $0.000,0.008,0.0001,0.013,0.0008$, respectively.

Acknowledgements The authors are thankful to Dr Aishwarya Venkataraman and the staff of NIH-NIRT-ICER laboratory at the National Institute for Research in Tuberculosis, Chennai for their help and support. The authors also express their sincere thanks to all the medical unit professors of ICH \& HC for treating children with PIMS-TS.

Authors' Contributions ES, PV were involved in concept development, data collection, analysis, and write up. PV, GK, GSV were involved in data collection, analysis, write up and review of literature. RSSJ was involved in concept development, analysis and review of literature. PV will act as the guarantor for this paper.

\section{Declarations}

Ethical Approval The study was approved by the Institutional Ethics Committee, Madras Medical College, Chennai.

Conflict of Interest None.

\section{References}

1. Lu X, Zhang L, Du H, et al. Chinese pediatric novel coronavirus study team. SARS-CoV-2 infection in children. N Engl J Med. 2020;382:1663-5.

2. Paediatric multisystem inflammatory syndrome temporally associated with COVID-19 (PIMS) - guidance for clinicians. In: RCPCH. 2020. Available at: https://www.rcpch.ac.uk/resources/paediatric-multisystem-inflammatory-syndrome-temporally-associated- covid-19-pimsguidance. Accessed on 3 Jan 2021.
3. CDC. Multisystem Inflammatory Syndrome in Children (MISC). In: Centers for Disease Control and Prevention. 2020. Available at: https://www.cdc.gov/mis-c/hcp/. Accessed on 1 Jan 2021.

4. Son MBF. Pediatric inflammatory syndrome temporally related to covid-19. BMJ. 2020;369:m2123.

5. Ramcharan T, Nolan O, Lai CY, et al. Paediatric inflammatory multisystem syndrome: temporally associated with SARSCoV-2 (PIMS-TS): cardiac features, management and shortterm outcomes at a UK tertiary paediatric hospital. Pediatr Cardiol. 2020;41:1391-401.

6. Tschudy MM, Arcara KM, The Harriet Lane handbook: A Manual for Pediatric House Officers. $19^{\text {th }}$ edition. Philadelphia, PA: Elsevier/Mosby; 2012. p. 8.

7. Newburger JW, Takahashi M, Burns JC. Kawasaki disease. J Am Coll Cardiol. 2016;67:1738-49.

8. Whittaker E, Bamford A, Kenny J, et al. PIMS-TS Study Group and EUCLIDS and PERFORM Consortia. Clinical characteristics of 58 children with a pediatric inflammatory multisystem syndrome temporally associated with SARS-CoV- 2. JAMA. 2020;324:259-69.

9. Dhanalakshmi K, Venkataraman A, Balasubramanian S, et al. Epidemiological and clinical profile of pediatric inflammatory multisystem syndrome - temporally associated with SARS-CoV-2 (PIMS-TS) in Indian children. Indian Pediatr. 2020;57:1010-4.

10. Williams V, Dash N, Suthar R, et al. Clinico-laboratory profile, intensive care needs, treatment details, and outcome of Pediatric inflammatory multisystem syndrome temporally associated with SARS-CoV-2 (PIMS-TS): a systematic review and metaanalysis. Journal of Pediatric Intensive Care. 2020. https://doi. org/10.1055/s-0040-1719173.

11. Paediatric inflammatory multisystem syndrome and SARS$\mathrm{CoV}-2$ infection in children . European Centre for Disease Prevention and Control. ECDC: Stockholm;2020. p. 7.

12. Cheung EW, Zachariah P, Gorelik M, et al. Multisystem inflammatory syndrome related to COVID-19 in previously healthy children and adolescents in New York City. JAMA. 2020;324:294-6.

13. Kaushik S, Aydin SI, Derespina KR, et al. Multisystem inflammatory syndrome in children associated with severe acute respiratory syndrome coronavirus 2 infection (MIS-C): a multi-institutional study from New York City. J Pediatr. 2020;224:24-9.

14. Belhadjer Z, Méot M, Bajolle F, et al. Acute heart failure in multisystem inflammatory syndrome in children in the context of global SARS- CoV-2 pandemic. Circulation. 2020;142:429-36.

15. Miller J, Cantor A, Zachariah P, Ahn D, Martinez M, Margolis KG. Gastrointestinal symptoms as a major presentation component of a novel multisystem inflammatory syndrome in children that is related to coronavirus disease 2019: a single center experience of 44 cases. Gastroenterology. 2020;159:1571-4.

16. Godfred-Cato S, Bryant B, Leung J, et al. COVID-19-Associated multisystem inflammatory syndrome in children-United States, march-july 2020. MMWR Morb Mortal Wkly Rep. 2020;69:1074-80.

17. Moraleda C, Serna-Pascual M, Soriano-Arandes A, et al. EPICO-AEP Working Group. Multi-inflammatory syndrome in children related severe acute respiratory syndrome coronavirus 2 (SARS-CoV-2) in Spain. Clin Infect Dis. 2021;72:397-401.

18. Nijman RG, De Guchtenaere A, Koletzko B, et al. Pediatric inflammatory multisystem syndrome: statement by the pediatric section of the European society for emergency medicine and European academy of pediatrics. Front Pediatr. 2020;8:490.

19. Riollano-Cruz M, Akkoyun E, Briceno-Brito E, et al. Multisystem inflammatory syndrome in children related to COVID-19: a New York City experience. J Med Virol. 2021;93:424-33. 
20. Feldstein LR, Rose EB, Horwitz SM, et al. Multisystem Inflammatory Syndrome in U.S. Children and Adolescents. In: New England Journal of Medicine. 2020. Available at: https://doi. org/10.1056/NEJMoa2021680. Accessed on 26 Nov 2020.

21. Jain S, Sen S, Lakshmivenkateshiah S, et al. Multisystem inflammatory syndrome in children with COVID-19 in Mumbai. India Indian Pediatr. 2020;57:1015-9.

22. White M, Tiesman B, Handforth J, Kenny J. Evelina PIMS TS working group. Paediatric inflammatory multisystem syndrometemporally associated with SARS-CoV-2 (PIMS-TS): the evelina experience. Arch Dis Child. 2020;105:1025-7.
23. Lawrensia S, Henrina J, Wijaya E, Suciadi LP, Saboe A, Cool CJ. Pediatric inflammatory multisystem syndrome temporally associated with SARS-CoV-2: a new challenge amid the pandemic. SN Compr Clin Med. 2020. https://doi.org/10.1007/ s42399-020-00602-8.

Publisher's note Springer Nature remains neutral with regard to jurisdictional claims in published maps and institutional affiliations. 